\title{
Serial dependence in position occurs at the time of perception
}

\author{
Mauro Manassi ${ }^{1}$ (i) $\cdot$ Alina Liberman ${ }^{1} \cdot$ Anna Kosovicheva ${ }^{2} \cdot$ Kathy Zhang ${ }^{1} \cdot$ David Whitney $^{1,3,4}$ \\ Published online: 26 March 2018 \\ (C) Psychonomic Society, Inc. 2018
}

\begin{abstract}
Observers perceive objects in the world as stable over space and time, even though the visual experience of those objects is often discontinuous and distorted due to masking, occlusion, camouflage, or noise. How are we able to easily and quickly achieve stable perception in spite of this constantly changing visual input? It was previously shown that observers experience serial dependence in the perception of features and objects, an effect that extends up to 15 seconds back in time. Here, we asked whether the visual system utilizes an object's prior physical location to inform future position assignments in order to maximize location stability of an object over time. To test this, we presented subjects with small targets at random angular locations relative to central fixation in the peripheral visual field. Subjects reported the perceived location of the target on each trial by adjusting a cursor's position to match its location. Subjects made consistent errors when reporting the perceived position of the target on the current trial, mislocalizing it toward the position of the target in the preceding two trials (Experiment 1). This pull in position perception occurred even when a response was not required on the previous trial (Experiment 2). In addition, we show that serial dependence in perceived position occurs immediately after stimulus presentation, and it is a fast stabilization mechanism that does not require a delay (Experiment 3). This indicates that serial dependence occurs for position representations and facilitates the stable perception of objects in space. Taken together with previous work, our results show that serial dependence occurs at many stages of visual processing, from initial position assignment to object categorization.
\end{abstract}

Keywords Serial effects $\cdot$ Sequential effects $\cdot$ Perceptual stability

Localization is one of the fundamental purposes of vision. Whether hunting for prey, looking for our wallet, or simply finding the computer mouse on our desk, correctly spotting an object's location is essential to everyday life. Localization has been shown to depend on several factors, including motion (De Valois \& De Valois, 1991; Ramachandran \& Anstis, 1990; Whitney \& Cavanagh, 2003; see also Whitney, 2002, for a review), spatial attention (Kerzel, 2000; Suzuki \& Cavanagh,

Mauro Manassi

mauro.manassi@berkeley.edu

1 Department of Psychology, University of California, Berkeley, CA, USA

2 Department of Psychology, Northeastern University, Boston, MA, USA

3 Helen Wills Neuroscience Institute, University of California, Berkeley, CA, USA

4 Vision Science Group, University of California, Berkeley, CA, USA
1997), frames of reference (Bridgeman, Peery, \& Anand, 1997), and eye movements (Cai, Pouget, Schlag-Rey, \& Schlag, 1997; Ross, Morrone, \& Burr, 1997; Ross, Morrone, Goldberg, \& Burr, 2001). Perceived position can also be influenced by stimulus history. For example, adaptation to location (e.g., adaptation to information such as luminance distribution or textures that define object position) and adaptation to motion can shift the perceived location of a subsequent stimulus (Bressler \& Whitney, 2006; McGraw, Whitaker, Skillen, \& Chung, 2002; Nishida \& Johnston, 1999; Snowden, 1998; Whitaker, McGraw, \& Levi, 1997; Whitney \& Cavanagh, 2003). These negative position aftereffects could reflect a mechanism that maximizes the visual system's sensitivity to change (Gepshtein, Lesmes, \& Albright, 2013).

Although sensitivity to change is clearly a useful function of vision, oversensitivity to change may not be a universally desirable state; if sensitivity were too high, it might lead to a jittery or unstable perception of object position. Moreover, the world around us is generally static and autocorrelated: objects tend to remain in the same locations over time. The visual system may therefore balance the need to maximize sensitivity 
to change with the likelihood that the world is relatively stable, and such a mechanism could facilitate the stable perception of object location.

Recent work has hypothesized a novel mechanism for object stabilization, suggesting that perception occurs through continuity fields: spatiotemporally tuned operators within which similar features and objects are integrated (Fischer \& Whitney, 2014). Continuity fields operate by inducing serial dependence in perception, making similar (but distinct) objects in time appear more similar than they actually are, and thus promoting the perception of object stability. For example, perceived orientation is systematically attracted toward previously seen orientations (Fischer \& Whitney, 2014). Serial dependence has been shown to shape the perception of a variety of other objects and features beyond simply orientation (Fischer \& Whitney, 2014), including faces (Liberman, Fischer, \& Whitney 2014; Taubert, Alais, \& Burr, 2016), attractiveness (Kondo, Takahashi, \& Watanabe, 2012; Taubert, Van der Burg, \& Alais, 2016; Xia, Leib, \& Whitney, 2016), ambiguous objects (Tafazoli, Di Filippo, \& Zoccolan, 2012; Wexler, Duyck, \& Mamassian, 2015), motion (Alais, Leung, \& Van der Burg, 2017), ensemble coding of orientation (Manassi, Liberman, Chaney, \& Whitney, 2017), numerosity (Cicchini, Anobile, \& Burr, 2014; Corbett, Fischer, \& Whitney, 2011), and has also been shown to support stable object identity perception when an object moves behind an occluder (Liberman, Zhang, \& Whitney 2016).

It remains unknown whether continuity fields only affect feature and object information or operate also on spatial information. Here, we tested whether continuity fields can generate serial dependence in the perceived position of objects. We flashed a target grating at random iso-eccentric locations and asked observers to report its position. We found systematic mislocalizations of the target, such that its reported location of the grating was biased: mislocalized toward previous target locations observed up to 10 seconds in the past.

\section{Method}

\section{Participants}

Participants were affiliates of UC Berkeley and provided written informed consent before participation. All participants had normal or corrected-to-normal vision, and all except two were naïve to the purpose of the experiment. Twelve subjects (six female) participated in Experiment 1, ranging in age from 19 to 33 years (mean $=25.5, S D=5$ ). Sixteen subjects (seven female) participated in Experiment 2, ranging in age from 19 to 34 years $($ mean $=25, S D=6$ ). Four subjects from Experiment 1 participated in Experiment 2. Twelve subjects (four female) participated in Experiment 3a, ranging in age from 18 to 34 years (mean $=23, S D=6$ ), and 11 subjects (five female) participated in Experiment $3 \mathrm{~b}$, ranging in age from 19 to 33 years (mean $=22, S D=4)$. All experimental procedures were approved by the UC Berkeley Institutional Review Board and were in accordance with the Declaration of Helsinki.

\section{Stimuli and procedure}

Experiments were conducted in a darkened experimental booth. Subjects viewed stimuli on a CRT monitor $(1024 \times$ 768, $100 \mathrm{~Hz}$, Dell Trinitron) at a distance of $56 \mathrm{~cm}$. All experiments were programmed in MATLAB (The MathWorks, Natick, MA) with Psychophysics Toolbox (Brainard, 1997).

Subjects were instructed to continuously fixate a central black dot $\left(0.21^{\circ}\right.$ in diameter $)$ on a gray background $(21 \mathrm{~cd} /$ $\mathrm{m}^{2}$ ) during the experiment. Observers were presented with a series of target stimuli (linear grating inside a circular aperture) at random locations on an iso-eccentric circle $\left(10^{\circ} \mathrm{ec}-\right.$ centricity; see Fig. 1a). Each target stimulus consisted of a vertically oriented static sine wave grating (four cycles per degree, Michelson contrast 30\%) embedded in a circular mask with a $3^{\circ}$ diameter (hard aperture). In order to increase difficulty in detecting the position of the Gabor, pink noise (1/f) was added to the grating. The position of the grating was randomized across 48 possible positions, in rotation steps of $7.5^{\circ}$ (see Fig. 1b). On each trial, the target was shown for 80 $\mathrm{ms}$, followed by a pink noise mask $(150 \mathrm{~ms})$ that filled the entire screen. The purpose of the mask was to minimize any possible negative aftereffect from the grating. Next, a black $\operatorname{dot}\left(0.5^{\circ}\right.$ diameter) appeared in a random location on the isoeccentric circle, also at $10^{\circ}$ eccentricity. Subjects were asked to adjust the black dot's position with the mouse to match the position of the grating. The dot was constrained to only move clockwise and counterclockwise along the invisible isoeccentric circle. The next trial began after an intertrial interval (ITI) of $2500 \mathrm{~ms}$. A long ITI was used in order to maintain a similar trial duration to Fischer and Whitney (2014). The fixation dot was present for the entire duration of the trial, including the ITI. Each observer completed 450 trials in total.

Experiment 2 was the same as Experiment 1, except that in $33 \%$ of the trials, observers were not asked to perform the position adjustment task. Rather, observers were presented with an interstimulus interval (ISI) of $1250 \mathrm{~ms}$, in which only the fixation dot was shown. The purpose of this experiment was to remove the response requirement and investigate whether motor response biases might play a role in any potential serial position effect. Each observer completed 1,380 trials in total.

Experiments $3 \mathrm{a}$ and $3 \mathrm{~b}$ were the same as Experiment 1, except that no mask was presented after the grating stimulus. In Experiments 3a and 3b, the gratings had a Michelson contrast of $30 \%$ and $4 \%$, respectively. 


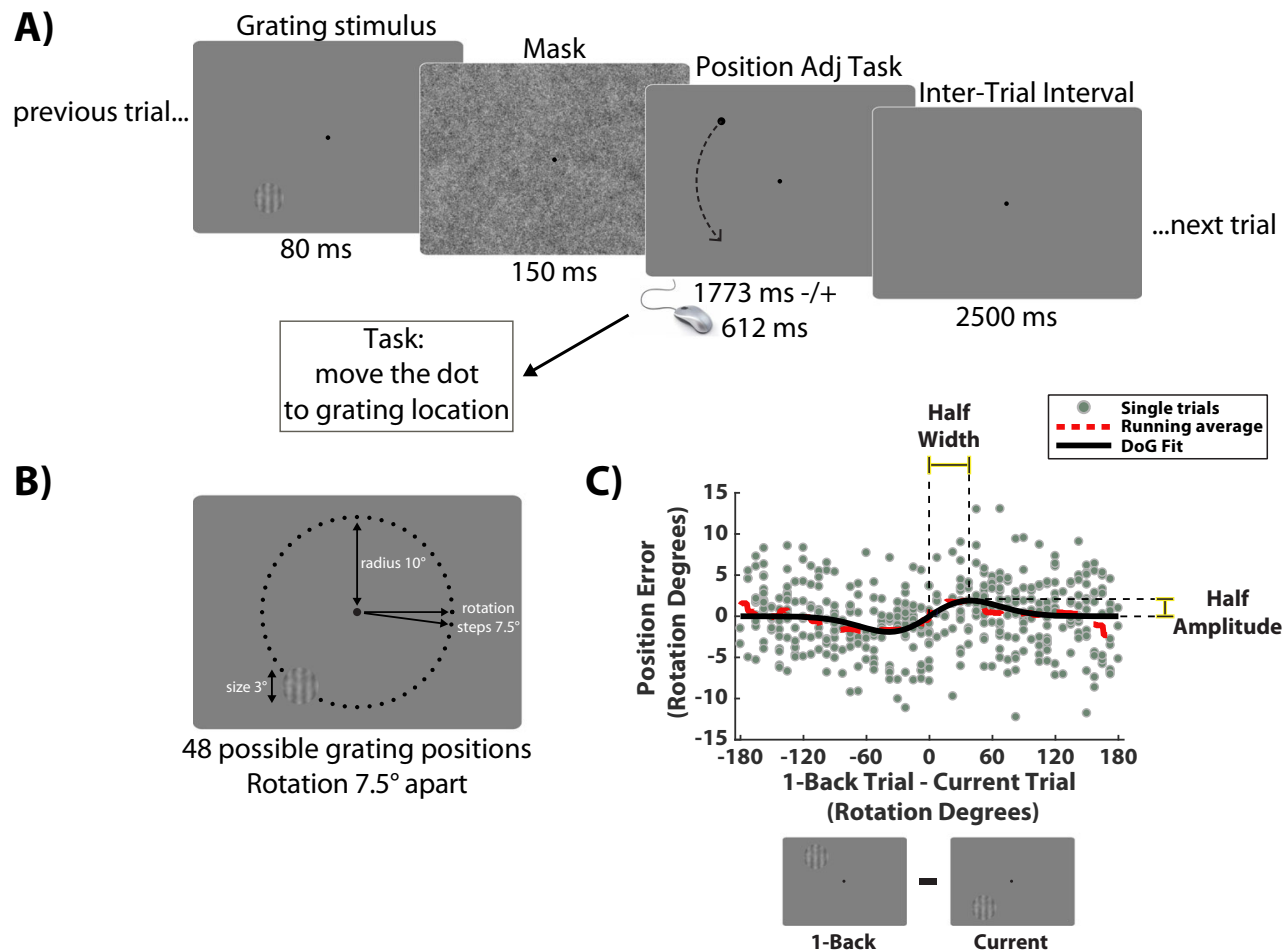

Fig. 1 Trial sequence in Experiment 1. a Observers were instructed to fixate on central dot during experiment. Target grating was presented at random location on invisible iso-eccentric circle for $80 \mathrm{~ms}$. After a noise mask of $150 \mathrm{~ms}$ (to reduce afterimages), subjects were asked to report the perceived location of the target by adjusting a dot's position to match the location of the grating. Trials were separated by a $2500-\mathrm{ms}$ delay. b A grating $\left(3^{\circ}\right.$ diameter) was presented at random angular locations relative to central fixation, at an eccentricity of $10^{\circ}$. There were 48 possible positions, in rotation steps of $7.5^{\circ}$. $\mathrm{c}$ Example data from a representative subject (1 trial back). Each data point shows performance on one trial.

\section{Data analysis}

For each subject's data, trials were considered lapses and excluded if the error exceeded 3 standard deviations from the grand mean error in perceived position, calculated for each individual subject, or if the response time (RT) was longer than $10 \mathrm{~s}$ (less than 5\% of data excluded on average). Response error for each trial was computed as the angular difference between the subject's response, given by the position of the adjustment dot and the actual position of the grating (see Fig. 1c; $y$-axis; reported target position minus actual target position). Position was always determined by the rotation angle from the fixation point at the center of the screen. Negative and positive values indicate that the subject's response was more counterclockwise or clockwise relative to the actual grating, respectively. Response error was then compared to the difference between the current and previous grating position (see Fig. 1c, $x$-axis; target position on previous trial minus target position on current trial). Negative and positive values indicate that the previous grating was in a more counterclockwise or clockwise position compared to the current grating, respectively.

The $x$-axis represents the difference between the previous position 1 trial back and the current position. The $y$-axis represents the error in the adjustment task (difference between dot position in the adjustment task and grating position on current trial). The average error (dashed line) shows more negative (counterclockwise) response errors for a negative relative position and more positive (clockwise) errors for a positive relative position. To quantify the magnitude of serial dependence, we fit a derivative of Gaussian (DoG) to the data (solid line) measuring the halfamplitude peak for each observer. (Color figure online)

In order to quantify the strength of serial dependence, we fit a simplified Gaussian derivative (DoG) to each subject's data (see Fig. 1c) of the form:

$y=a b c x e^{-(b x)^{2}}$,

where $y$ is response error on each trial, $x$ is the relative orientation of the previous trial, $a$ is half the peak-to-trough amplitude of the derivative-of-Gaussian, $b$ scales the width of the Gaussian derivative, and $c$ is a constant $\sqrt{2} / \mathrm{e}^{-0.5}$, which scales the curve to make the $a$ parameter equal to the peak amplitude. We fit the Gaussian derivative using constrained nonlinear minimization of the residual sum of squares. As a measure of serial dependence, we report half the peak-totrough amplitude (parameter $a$; see Figs. 2a, 3, 4) and half the width (parameter $b$; see Fig. 2b) of the best fitting derivative of Gaussian. A positive value for the $a$ parameter indicates a perceptual bias toward the position of the previous grating. A negative value for the $a$ parameter indicates a perceptual bias away from the position of the previous grating. A value of zero for the $a$ parameter indicates no bias. 


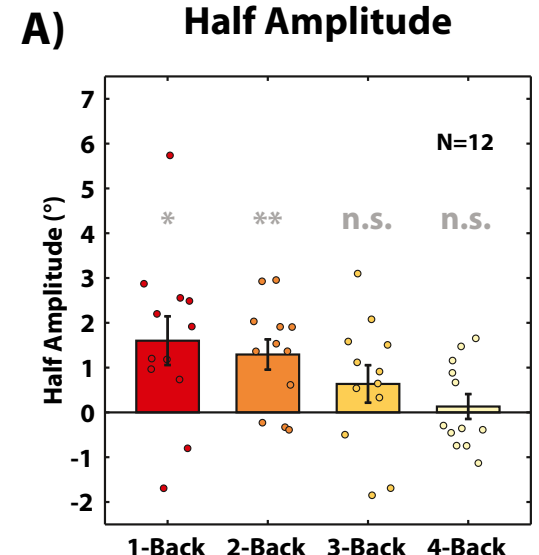

Fig. 2 Experiment 1. Average half amplitudes (a) and widths (b) of derivative of Gaussian fit across 12 subjects for 1,2, 3, and 4 trials back. Each filled dot represents the half amplitude or width for a single

Kosovicheva and Whitney (2017) recently showed that that individual subjects have idiosyncratic biases in reported position unrelated to serial dependence, such as perceptual distortions at different points around the iso-eccentric stimulus circle. For this reason, we conducted an additional control analysis to remove such potential unrelated biases before fitting the Gaussian derivative function described in Equation 1. To do this, we fit a polynomial function (10 degrees) to each observer's error distribution (reported target position minus actual target position) as a function of target location on the circle. Systematic motor error, for example, might manifest as a bias to consistently report a target presented at the 12 o'clock position as being at the 2 o'clock position. To regress out such biases, we subtracted the subject's reported target positions from the discretized polynomial fit. This subtraction left us with residual errors that did not include the idiosyncratic biases unrelated to serial dependence. We then plotted these residual errors as a function of the difference between current and previous target location ( $x$-axis in Fig. 1c) and fit the Gaussian derivative to the data. Importantly, the addition of this control analysis - removing systematic biases unrelated to serial effects - had no significant impact on the serial dependence results. It did not generate or increase the measured serial dependence.

\section{Results}

\section{Experiment 1: Serial dependence in perceived position}

Ten subjects out of 12 displayed a positive DoG half amplitude, indicating that perceived position on a given trial was significantly pulled in the direction of grating position on the preceding (i.e., 1-back) trial, $t(11)=2.94, p=0.013$ (see Fig. $2 a)$. Even when comparing subjects' errors with the difference

\section{B) Half Width}

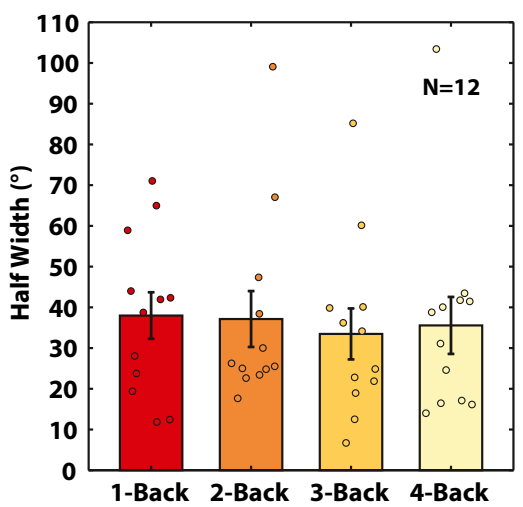

subject (horizontal jitter added for visibility). Bars indicate the average and error bars indicate \pm one standard error. Asterisks represent a significant difference from zero. (Color figure online)

in position two trials back, subjects showed significantly positive DoG half amplitudes, $t(11)=3.82, p<.01$ (see Fig. 2a), meaning that even grating positions presented two trials back biased perceived position on a given trial. For three and four trials back, DoG half amplitudes were not significantly different from zero: 3-back, $t(11)=1.51, p=.15$; 4-back, $t(11)=$ $0.47, p=.64$ (see Fig. 2a). Average response time across subjects was $1773 \pm 612 \mathrm{~ms}$. The perceived position of the grating was therefore strongly attracted toward previous grating positions seen 5 seconds (one trial back, Fig. 2a) or 10 seconds ago (two trials back, Fig. 2a).

We also analyzed the width of the DoG fit in order to address whether the temporal tuning of serial dependence (1-2 trials back) determines its spatial tuning (i.e., the width in the DoG fit). There were no statistically significant differences in width between 1,2,3, and 4 trials back as determined

\section{Half Amplitude} (1-Back)

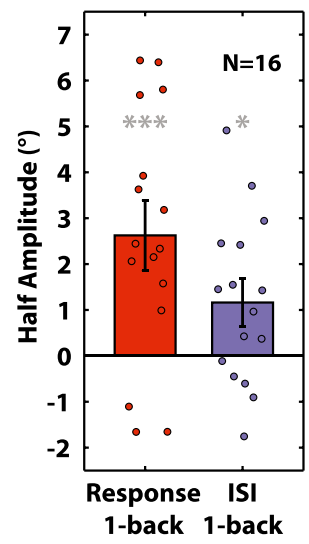

Fig. 3 Experiment 2. Average half amplitudes of derivative of Gaussian when previous trial required a response (previous response, left bar) and when previous trial was interleaved with an interstimulus interval of $1250 \mathrm{~ms}$ (no response, right bar). (Color figure online) 
A)

Experiment 3A (High Contrast)

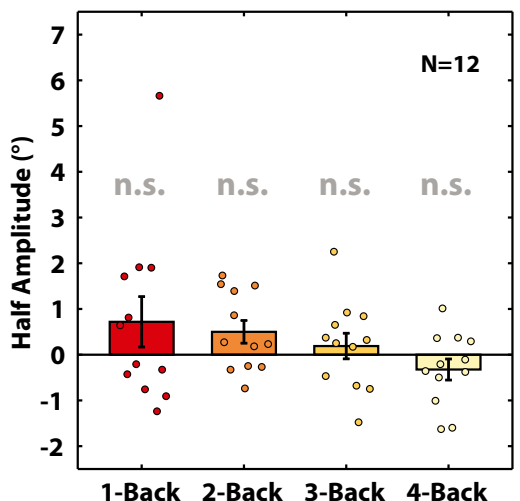

Fig. 4 Experiment $3 \mathrm{a}$ and $3 \mathrm{~b}$. Average half amplitudes of derivative of Gaussian fit for 1, 2, 3, and 4 trials back. Grating contrast was 30\% in Experiment $3 \mathrm{a}$ and $4 \%$ in Experiment $3 \mathrm{~b}$. Each filled dot represents the

by a one-way ANOVA, $F(3,44)=0.09, p=.96$. In addition, there was no correlation between half amplitudes and half width (see Fig. 2b; $r=-0.16, p=.26$ ).

\section{Experiment 2: Serial dependence in position is not due to previous motor response}

In Experiment 1, we found that subjects made consistent errors when reporting the perceived location of the target grating on the current trial, mislocalizing it toward the location presented on the previous two trials.

In Experiment 2, we tested whether this position bias may be due to the previous motor response. In this experiment, on $33 \%$ of the trials, the position adjustment task was replaced with an ISI of $1250 \mathrm{~ms}$. Importantly, position adjustment task trials (67\% of trials) and no-task trials (33\% of trials) were presented in a random fashion, and observers did not know whether they would have been asked to respond or not to the target in a given trial. If serial dependence observed in Experiment 1 was due to the previous motor response, then we should expect no evidence of serial dependence in the position adjustment task if it was preceded by a trial containing an ISI in place of the position adjustment task. Each subject completed 1380 trials divided into 12 blocks.

In both sequences (see Fig. 3), subjects displayed on average positive DoG half amplitudes, previous response: $t(15)=$ $3.94, p<.001$; no previous response: $t(15)=2.58, p=.02$, meaning that serial dependence in perceived position did not require a previous motor response (see Fig. 3, left and right bars). We also found increased serial dependence when the previous trial required a response (see Fig. 3, left bar) than when it did not (see Fig. 3, right bar), $t(15)=3.87, p<.001$. This increase in effect observed in the response conditions was likely due to the fact that subjects were presented with

\section{B) Experiment 3B (Low Contrast)}

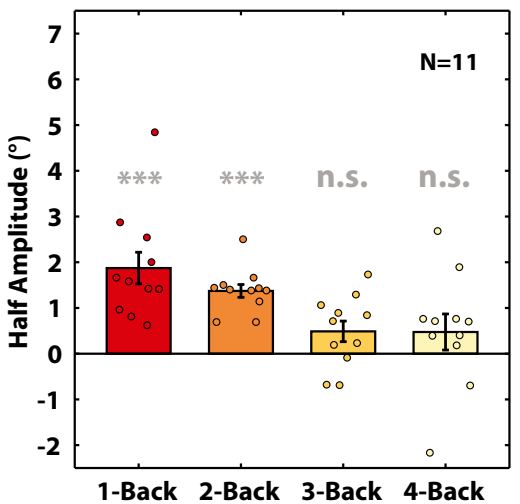

half amplitude for a single subject. Bars indicate the average and error bars indicate \pm one standard error. The asterisks represent a significant difference from zero. (Color figure online)

an additional dot at the same grating position, thus reinforcing the serial dependence effect.

As an alternative analysis, we could have measured serial dependence from the previous response by computing the $x$-axis (see Fig. 1c) as "adjusted dot position in previous trial (previous response rather than previous stimulus) minus grating position on current trial." However, in this specific analysis, motor response biases (Shaffer, 1978; Wing \& Kristofferson, 1973), oblique effects (Appelle, 1972; Cicchini, Mikellidou, \& Burr, 2017; Mikellidou, Cicchini, Thompson, \& Burr, 2015), or other localization biases (e.g., Kosovicheva \& Whitney, 2017) can be consistent and therefore correlate across trials. Any of those consistent biases can create artifacts that resemble serial dependence, but are in fact unrelated. Because of these methodological issues, previous studies have measured serial dependence between current and previous stimuli (Cicchini et al., 2017; Fischer \& Whitney, 2014; Liberman et al., 2014; Manassi et al., 2017), rather than comparing previous and current response.

\section{Experiment 3a and 3b: Fast serial dependence in position perception}

Recently, it was proposed that serial dependence in position perception is a phenomenon of mnemonic rather than perceptual processes (Bliss, Sun, \& D'Esposito, 2017). With a very similar paradigm ${ }^{1}$, the authors varied the delay between stimulus presentation (a very high contrast black dot) and response (dot position adjustment) and found that positive serial dependence occurred for delays between the target dot and the adjustment dot that ranged from 1 to 10 seconds. Interestingly, when there was no delay between the target and adjustment dot

\footnotetext{
${ }^{1}$ Their work was independently submitted after our initial submission to the Psychonomic Bulletin \& Review.
} 
(and no mask), there was a negative aftereffect instead of positive serial dependence (see Fig. 2b in Bliss et al., 2017). It is known that positive serial dependence and adaptation-induced negative aftereffects can be additive (Alais et al., 2017; Cicchini et al., 2017; Fischer \& Whitney, 2014; Taubert, Alais, et al., 2016), and the negative aftereffect Bliss et al. (2017) report could be the result of adaptation to the high contrast, salient target dot, as previous authors have reported (Hess, Dakin, \& Badcock, 1994; Whitaker et al., 1997). To reduce adaptation and negative aftereffects, Experiment 1 used a mask following the stimulus. In Experiments $3 \mathrm{a}$ and $3 \mathrm{~b}$, we removed the mask and varied the contrast of the target in order to test whether serial dependence occurs when there is zero delay between stimulus presentation and response.

Experiment 3a was the same as Experiment 1, except that no noise mask was presented after the target grating presentation. The noise mask was removed in order to keep the interval between target and response stimulus at zero. Michelson contrast of the grating was kept at $30 \%$ as in Experiments 1 and 2 (high contrast experiment). Each observer completed 450 trials in total.

Subjects showed no evidence of serial dependence or negative aftereffect. The DoG half amplitudes were not significantly different from zero for any trial back, 1-back: $t(11)=1.30, p=$ 0.21 ; 2-back: $t(11)=2.01, \mathrm{p}=0.07$; 3-back: $t(11)=0.67, \mathrm{p}=$ 0.51 ; 4-back: $t(11)=-1.40, \mathrm{p}=0.18$ (see Fig. 4a). Taken together, these results are in accordance with previous results (Bliss et al., 2017), showing no serial dependence for a 0 delay between stimulus and response (Experiment 3a), and serial dependence for longer delays (Experiments 1-2). However, this does not mean that the delay, per se, is the critical factor.

The critical factor that modulates serial dependence in perceived position may be the effective contrast of the target stimulus. Previous evidence showed that the perceived contrast of a grating can be weakened by a subsequent (backward) mask (Breitmeyer, Rudd, \& Dunn, 1981; Breitmeyer, Hoar, Randall, \& Conte, 1984; Breitmeyer \& Ogmen, 2000; Kolers, 1962; Raab, 1963) and, as a consequence, its negative aftereffect is weakened (Gibson \& Radner, 1937; Keck, Palella, \& Pantle, 1976; Stecher, Sigel, \& Lange, 1973). Accordingly, a grating with a backward mask showed serial dependence (Experiments 1 and 2), whereas a grating without mask showed no evidence of serial dependence (Experiment 3a). Hence, if the apparent reduction in target contrast (and not the delay) determines the strength of serial dependence, we hypothesized that serial dependence should arise with a lower contrast grating. In Experiment 3b, we tested this hypothesis.

In Experiment 3b, we reduced the Michelson contrast of the grating to $4 \%$ (low contrast experiment). Our aim was twofold. First, by reducing contrast, any adaptation induced negative aftereffect (Hess et al., 1994; McGraw et al., 2002; Whitaker et al., 1997) would be reduced. Second, subjects are forced to pay more attention to the target grating. As serial dependence strongly depends on attention (Fischer \& Whitney, 2014), a stronger positive bias is expected.

In accordance with our hypothesis, subjects showed evidence of serial dependence, even with zero delay between the target and response stimulus. This held for one and two trials back, 1-back: $t(10)=5.18, p<.001 ; 2$-back: $t(10)=9.36, p<$ .001 ; 3-back: $t(10)=2.07, p=.06$; 4-back: $t(10)=1.15, p=$ .27 (see Fig. 4b). Hence, the critical factor that determines the strength of serial dependence in localization judgments is the luminance contrast (or uncertainty more generally), not the delay. Serial dependence in position is a fast mechanism, biasing position perception immediately after stimulus presentation. Although serial dependence may still be modulated by short-term working memory (Bliss et al., 2017; Fritsche, Mostert, \& de Lange, 2017), it cannot be considered a purely delay-dependent or working-memory-based process detached from its perceptual component (see also Cicchini et al., 2017).

\section{Discussion}

The world around us appears stable despite changes in noise and lighting, discontinuities such as eye blinks, and changes in gaze and head position. Previous studies have proposed that, in order to facilitate perceptual stability, perception occurs through continuity fields: spatiotemporal regions over which object are attracted toward recently seen objects and features (Fischer \& Whitney, 2014; Liberman et al., 2014). On a neural level, continuity fields may advantageously reduce cortical processing and reprocessing from moment to moment, by recycling representations of previously perceived features and objects. On a perceptual level, continuity fields help observers maintain a continuous and stable representation of the world.

Recent studies have also suggested that there is serial dependence across species. Papadimitriou, Ferdoash, and Snyder (2015) found that when monkeys made saccades to remembered positions, the saccades were biased toward previous saccade targets. Along the same lines, Tafazoli et al. (2012) found that when rats were trained to learn the appearance of a default object, they perceived two new subsequent objects as similar to the previous default one. Our results are consistent with and extend these prior studies, showing that serial dependence occurs in human spatial vision, even in tasks as fundamental as perceptual localization.

To summarize our findings across the experiments reported here, we found (a) a positive aftereffect in position: position perception was pulled by object positions encountered 5 or 10 seconds ago (Experiment 1; Fig. 2). (b) This kind of serial dependence is not due to the subject's motor response in the previous trial; when we eliminated the motor response in the 1-back trial, we still found a significant serial dependence effect on position in the current trial (Experiment 2; Fig. 3). 
(c) Serial dependence in position perception occurs immediately, independent on the delay between stimulus and response (Experiment 3a and 3b; Fig. 4). Taken together, our results suggest that serial dependence can occur in position representations, providing a mechanism through which continuity fields can promote the appearance of position consistency from moment to moment.

Our results may involve serial dependence at the level of spatial memory representations (Papadimitriou et al., 2015; Rahnev, Koizumi, McCurdy, D’Esposito, \& Lau, 2015). In fact, there may be a strong connection between serial dependence in perception and in memory (Kiyonaga, Scimeca, Bliss, \& Whitney, 2017; see also Makovski \& Jiang, 2008; Papadimitriou et al., 2015). Serial dependence has a clear perceptual component (Cicchini et al., 2017; Fischer \& Whitney, 2014), but higher level factors like decision (de Lange \& Fritsche, 2017; Fritsche et al., 2017) and memory (Bliss et al., 2017) may still play a role in determining its strength. It is also possible that there is serial dependence at multiple levels of representation, including at basic perceptual levels (Cicchini et al., 2017; Fischer \& Whitney, 2014), perceptual decisions (Fritsche et al., 2017), and also in memory representations (Papadimitriou et al., 2015; Zhang, Liberman, \& Whitney, 2016). How these multiple levels interact remains an exciting area of investigation.

Prior work has shown that previous trials can affect subsequent trials in detecting target position (Maljkovic \& Nakayama, 1996) through priming. However, this priming effect manifests primarily as a reduction in reaction times: Reaction times decreased when the target position was repeated on consecutive trials and increased when the target fell on a position previously occupied by a distractor. Conversely, our results showed that previous positions biased the reported location of the target. While it is still debated whether serial effects are due to a change in perception or decision, continuity fields underlie all these effects for the same purpose: to promote stable localization in the complex environments we experience everyday (Fischer \& Whitney, 2014; Manassi et al., 2017).

Previous work on perceptual localization has shown that adaptation to motion (McGraw et al., 2002; Nishida \& Johnston, 1999; Snowden, 1998; Whitaker et al., 1997; Whitney, 2005; Whitney \& Cavanagh, 2003) and luminance- or texture-defined stimuli (Hess et al., 1994; McGraw et al., 2002; Nishida \& Johnston, 1999; Snowden, 1998; Whitaker et al., 1997) can lead to a repulsion effect, biasing perceived visual position away from previously seen adaptors. Our results reveal an attraction toward previous positions. The visual system implements adaptation and repulsion mechanisms in order to maximize sensitivity to change or differences. Our results indicate that there is a complementary attraction mechanism that facilitates perceptual stability of position. These two mechanisms could be opposite sides of the same coin: both facilitate constancy and stability, but do so differently. Open questions remain as to how adaptation (and repulsion effects more generally) interact with serial dependence, and some work has begun to investigate this (Fischer \& Whitney, 2014; Taubert, Alais, et al., 2016). For example, there may be different time courses for adaptation and serial dependence, or a different dependence on noise, interstimulus intervals, or storage, among other factors. Future research should investigate the interplay between these two opposing mechanisms and how the balance between these processes facilitates our perception of stability.

In conclusion, our results provide evidence that serial dependence occurs for object localization. Together with previous work, this suggests that continuity fields determine visual perception at several stages of visual processing. Serial dependence has been shown to occur for low-level features, such as position assignment and orientation perception (Fischer \& Whitney, 2014; Fritsche et al., 2017; Liberman et al., 2016), as well as for high level features such as face perception (Liberman et al., 2014; Taubert, Alais, et al., 2016) and attractiveness (Kondo et al., 2012; Taubert, Van der Burg, et al., 2016; Xia et al., 2016). The existence of continuity fields at several stages of visual processing, along with their spatial and temporal tuning, suggests that the underlying neural mechanism(s) likely involves feedback, but is not likely to be a single stage process or a unitary decision effect.

Acknowledgements This work was supported in part by the Swiss National Science Foundation fellowship P2ELP3_158876 (M.M.) and NSF graduate research fellowships (NSF-GRFP) to A.K. and A.L. This work was originally presented at Vision Science Society Annual Meeting in 2014. We would like to thank Daniel Bliss for useful discussions.

\section{References}

Alais, D., Leung, J., \& Van der Burg, E. (2017). Linear summation of repulsive and attractive serial dependencies: orientation and motion dependencies sum in motion perception. Journal of Neuroscience, 37(16), 4381-4390. https://doi.org/10.1523/JNEUROSCI.4601-15. 2017

Appelle, S. (1972). Perception and discrimination as a function of stimulus orientation: The "oblique effect" in man and animals. Psychological Bulletin, 78(4), 266.

Bliss, D. P., Sun, J. J., \& D'Esposito, M. (2017). Serial dependence is absent at the time of perception but increases in visual working memory. Scientific Reports, 7(1), 14739. https://doi.org/10.1038/ s41598-017-15199-7

Brainard, D. H. (1997). The Psychophysics Toolbox. Spatial Vision, 10(4), 433-436. https://doi.org/10.1163/156856897X00357

Breitmeyer, B. G., Hoar, W. S., Randall, D., \& Conte, F. P. (1984). Visual masking: An integrative approach. London, UK: Clarendon Press.

Breitmeyer, B. G., \& Ogmen, H. (2000). Recent models and findings in visual backward masking: A comparison, review, and update. Perception \& Psychophysics, 62(8), 1572-1595.

Breitmeyer, B. G., Rudd, M., \& Dunn, K. (1981). Metacontrast investigations of sustained-transient channel inhibitory interactions. Journal of Experimental Psychology: Human Perception and 
Performance, 7(4), 770-779. https://doi.org/10.1037/0096-1523.7. 4.770

Bressler, D. W., \& Whitney, D. (2006). Second-order motion shifts perceived position. Vision Research, 46(6/7), 1120-1128. https://doi. org/10.1016/j.visres.2005.10.012

Bridgeman, B., Peery, S., \& Anand, S. (1997). Interaction of cognitive and sensorimotor maps of visual space. Perception \& Psychophysics, 59(3), 456-469. https://doi.org/10.3758/BF03211912

Cai, R. H., Pouget, A., Schlag-Rey, M., \& Schlag, J. (1997). Perceived geometrical relationships affected by eye-movement signals. Nature, 386(6625), 601-604. https://doi.org/10.1038/386601a0

Cicchini, G. M., Anobile, G., \& Burr, D. C. (2014). Compressive mapping of number to space reflects dynamic encoding mechanisms, not static logarithmic transform. Proceedings of the National Adacemy of Sciences of the United States of America, 111(21), 7867-7872. https://doi.org/10.1073/pnas.1402785111

Cicchini, G. M., Mikellidou, K., \& Burr, D. (2017). Serial dependencies act directly on perception. Journal of Vision, 17(14), 6. https://doi. org/10.1167/17.14.6

Corbett, J. E., Fischer, J., \& Whitney, D. (2011). Facilitating stable representations: Serial dependence in vision. PLOS ONE, 6(1), e16701. https://doi.org/10.1371/journal.pone.0016701

de Lange, F. P., \& Fritsche, M. (2017). Perceptual decision-making: Picking the low-hanging fruit? Trends in Cognitive Sciences, 21(5), 306-307. https://doi.org/10.1016/j.tics.2017.03.006

De Valois, R. L., \& De Valois, K. K. (1991). Vernier acuity with stationary moving Gabors. Vision Research, 31(9), 1619-1626. https://doi. org/10.1016/0042-6989(91)90138-U

Fischer, J., \& Whitney, D. (2014). Serial dependence in visual perception. Nature Neuroscience, 17(5), 738-743. https://doi.org/10.1038/nn. 3689

Fritsche, M., Mostert, P., \& de Lange, F. P. (2017). Opposite effects of recent history on perception and decision. Current Biology, 27(4), 590-595. https://doi.org/10.1016/j.cub.2017.01.006

Gepshtein, S., Lesmes, L. A., \& Albright, T. D. (2013). Sensory adaptation as optimal resource allocation. Proceedings of the National Adacemy of Sciences of the United States of America, 110(11), 4368-4373. https://doi.org/10.1073/pnas.1204109110

Gibson, J. J., \& Radner, M. (1937). Adaptation, after-effect and contrast in the perception of tilted lines: I. Quantitative studies. Journal of Experimental Psychology, 20(5), 453.

Hess, R. F., Dakin, S. R., \& Badcock, D. (1994). Localization of element clusters by the human visual system. Vision Research, 34(18), 2439 2451. https://doi.org/10.1016/0042-6989(94)90288-7

Keck, M. J., Palella, T. D., \& Pantle, A. (1976). Motion aftereffect as a function of the contrast of sinusoidal gratings. Vision Research, 16(2), 187-191. https://doi.org/10.1016/0042-6989(76)90097-3

Kerzel, D. (2000). Eye movements and visible persistence explain the mislocalization of the final position of a moving target. Vision Research, 40(27), 3703-3715. https://doi.org/10.1016/S00426989(00)00226-1

Kiyonaga, A., Scimeca, J. M., Bliss, D. P., \& Whitney, D. (2017). Serial dependence across perception, attention, and memory. Trends in Cognitive Sciences, 21(7), 493-497. https://doi.org/10.1016/j.tics. 2017.04.011

Kolers, P. A. (1962). Intensity and contour effects in visual masking. Vision Research, 2(9/10), 277-274.

Kondo, A., Takahashi, K., \& Watanabe, K. (2012). Sequential effects in face-attractiveness judgment. Perception, 41(1), 43-49. https://doi. org/10.1068/p7116

Kosovicheva, A., \& Whitney, D. (2017). Stable individual signatures in object localization. Current Biology, 27(14), R700-R701. https:// doi.org/10.1016/j.cub.2017.06.001

Liberman, A., Fischer, J., \& Whitney, D. (2014). Serial dependence in the perception of faces. Current Biology, 24(21), 2569-2574. https:// doi.org/10.1016/j.cub.2014.09.025
Liberman, A., Zhang, K., \& Whitney, D. (2016). Serial dependence promotes object stability during occlusion. Journal of Vision, 16(15), 16. https://doi.org/10.1167/16.15.16

Makovski, T., \& Jiang, Y. V. (2008). Proactive interference from items previously stored in visual working memory. Memory \& Cognition, 36(1), 43-52. https://doi.org/10.3758/MC.36.1.43

Maljkovic, V., \& Nakayama, K. (1996). Priming of pop-out: II. The role of position. Perception \& Psychophysics, 58(7), 977-991. https:// doi.org/10.3758/BF03206826

Manassi, M., Liberman, A., Chaney, W., \& Whitney, D. (2017). The perceived stability of scenes: Serial dependence in ensemble representations. Scientific Reports, 7(1), 1971. https://doi.org/10.1038/ s41598-017-02201-5

McGraw, P. V., Whitaker, D., Skillen, J., \& Chung, S. T. (2002). Motion adaptation distorts perceived visual position. Current Biology, 12(23), 2042-2047.

Mikellidou, K., Cicchini, G. M., Thompson, P. G., \& Burr, D. C. (2015). The oblique effect is both allocentric and egocentric. Journal of Vision, 15(8), 24-24.

Nishida, S., \& Johnston, A. (1999). Influence of motion signals on the perceived position of spatial pattern. Nature, 397(6720), 610-612. https://doi.org/10.1038/17600

Papadimitriou, C., Ferdoash, A., \& Snyder, L. H. (2015). Ghosts in the machine: Memory interference from the previous trial. Journal of Neurophysiology, 113(2), 567-577. https://doi.org/10.1152/jn. 00402.2014

Raab, D. H. (1963). Backward masking. Psychological Bulletin, 60(2), 118.

Rahnev, D., Koizumi, A., McCurdy, L. Y., D’Esposito, M., \& Lau, H. (2015). Confidence leak in perceptual decision making. Psychological Science, 26(11), 1664-1680. https://doi.org/10. 1177/0956797615595037

Ramachandran, V. S., \& Anstis, S. M. (1990). Illusory displacement of equiluminous kinetic edges. Perception, 19(5), 611-616. https://doi. org/10.1068/p190611

Ross, J., Morrone, M. C., \& Burr, D. C. (1997). Compression of visual space before saccades. Nature, 386(6625), 598.

Ross, J., Morrone, M. C., Goldberg, M. E., \& Burr, D. C. (2001). Changes in visual perception at the time of saccades. Trends Neurosci, 24(2), 113-121. https://doi.org/10.1016/S0166-2236(00) 01685-4

Shaffer, L. (1978). Timing in the motor programming of typing. The Quarterly Journal of Experimental Psychology, 30(2), 333-345.

Snowden, R. J. (1998). Shifts in perceived position following adaptation to visual motion. Current Biology, 8(24), 1343-1345. https://doi. org/10.1016/S0960-9822(07)00567-2

Stecher, S., Sigel, C., \& Lange, R. V. (1973). Spatial frequency channels in human vision and the threshold for adaptation. Vision Research, 13(9), 1691-1700. https://doi.org/10.1016/0042-6989(73)90088-6

Suzuki, S., \& Cavanagh, P. (1997). Focused attention distorts visual space: An attentional repulsion effect. Journal of Experimental Psychology: Human Perception and Performance, 23(2), 443463. https://doi.org/10.1037/0096-1523.23.2.443

Tafazoli, S., Di Filippo, A., \& Zoccolan, D. (2012). Transformationtolerant object recognition in rats revealed by visual priming. Journal of Neuroscience, 32(1), 21-34. https://doi.org/10.1523/ JNEUROSCI.3932-11.2012

Taubert, J., Alais, D., \& Burr, D. (2016). Different coding strategies for the perception of stable and changeable facial attributes. Scientific Reports, 6, 32239. https://doi.org/10.1038/srep32239

Taubert, J., Van der Burg, E., \& Alais, D. (2016). Love at second sight: Sequential dependence of facial attractiveness in an on-line dating paradigm. Scientific Reports, 6, 22740. https://doi.org/10.1038/ srep22740

Wexler, M., Duyck, M., \& Mamassian, P. (2015). Persistent states in vision break universality and time invariance. Proceedings of the 
National Adacemy of Sciences of the United States of America, 112(48), 14990-14995. https://doi.org/10.1073/pnas.1508847112

Whitaker, D., McGraw, P. V., \& Levi, D. M. (1997). The influence of adaptation on perceived visual location. Vision Research, 37(16), 2207-2216. https://doi.org/10.1016/S0042-6989(97)00030-8

Whitney, D. (2002). The influence of visual motion on perceived position. Trends in Cognitive Sciences, 6(5), 211-216. https://doi.org/10. 1016/S1364-6613(02)01887-9

Whitney, D. (2005). Motion distorts perceived position without awareness of motion. Current Biology, 15(9), R324-326. https://doi.org/ 10.1016/j.cub.2005.04.043
Whitney, D., \& Cavanagh, P. (2003). Motion adaptation shifts apparent position without the motion aftereffect. Perception \& Psychophysics, 65(7), 1011-1018. https://doi.org/10.3758/BF03194830

Wing, A. M., \& Kristofferson, A. B. (1973). Response delays and the timing of discrete motor responses. Perception \& Psychophysics, 14(1), 5-12.

Xia, Y., Leib, A. Y., \& Whitney, D. (2016). Serial dependence in the perception of attractiveness. Journal of Vision, 16(15), 28. https:// doi.org/10.1167/16.15.28

Zhang, K., Liberman, A., \& Whitney, D. (2016). Perceptual stability without working memory. Journal of Vision, 16, 1078. 